\title{
An Evaluation Method for Heart Rate Variability, by Using Acceleration Plethysmography
}

\author{
Haruko TAKADA ${ }^{1}$; Kazuo OKINO ${ }^{2}$; and Yumiko NIWA ${ }^{3}$ \\ ${ }^{I}$ College of Medical Engineering, Suzuka University of Medical and Science, ${ }^{2}$ Research Section, U-medica Inc. Co., and ${ }^{3}$ Clinical Examination, \\ Meitetsu Hospital
}

\begin{abstract}
Objects We have recently developed a new type of acceleration plethysmography (APG) machine and software. This APG system functions to indicate heart rate variability, by using the coefficient of the variation of the a-a intervals (CVaa\%). We tried to evaluate the validity of the APG system to estimate heart rate variability, instead of using ECG.

Methods We simultaneously recorded an ECG and an APG by using a 2-channel APG system. We examined the difference between the a-a intervals (Taa) of an APG and the R-R interval of an ECG. Next, APG waveforms of 121 healthy people and 26 diabetics were recorded, and the mean CVaa\% in each age category for healthy people and diabetics were calculated.
\end{abstract}

Results 1) All differences between the Taa and the R-R interval were within $10 \mathrm{msec}$, and the multiple correlation coefficient was 0.999175 .2 ) The mean CVaa\% was 0.2 to $1.5 \%$ bigger than the mean coefficient of the variation of the R-R intervals (CVRR\%) in all age categories. 3) The mean CVaa\% decreased by age. 4) In ages $40-60$, mean CVaa\% of diabetics was significantly lower than that of healthy people.

Conclusion The APG system is suitable to estimate heart rate variability. And, when we want to evaluate heart rate variability by $\mathrm{CVaa} \%$ of an APG in stead of CVRR\% of an ECG, a standard value of CVaa\% in each age is necessary. The APG system has some possibilities for explanation of a mechanism of autonomic-nerve-related diseases.

Key Words Acceleration Plethysmography, Aging, Autonomic nerve, Diabetes, Heart rate variability

\section{INTRODUCTION}

We have recently developed a new APG system. The new APG machine is small like a "mouse", and can be connected to a personal computer by USB port. We can easily measure APG waveforms from the fingertip of a subject in a sitting position. The software consists of three units to record, to measure and to analyze APG. When we record APG waveforms for 18 seconds by this system, it gives information about the degree of arteriosclerosis or "the relative vascular

Received: October 16, 2003

${ }^{1}$ Address; College of Medical Engineering, Suzuka University of Medical and Science, 1001-1, Kishioka, Suzuka City, Mie, 5100293 Japan. TEL: 81-593-83-9208 (ext. 2408), FAX: 81-593-839666, E-mail:

${ }^{2}$ Address; Research Section, U-medica Inc. Co., 5-5, Koubaicho, Kita-ku, Osaka City, 530-0038 Japan. TEL: 81-6-4800-8626, FAX: 81-6-4800-8627

${ }^{3}$ Address; Clinical Examination, Meitetsu Hospital, 2-26-11, Sakou, Nishi-ku, Nagoya City, 451-8511 Japan. TEL: 81-52-5516121 age" automatically. ${ }^{[1],[2]}$

This APG system also has another function. It gives information of heart rate variability, if we measure APG for two minutes. Heart rate variability can be estimated by the coefficient of the variation of the a-a intervals (CVaa\%) of an APG.

The heart rate variability had been obtained by the coefficient of the variation of the R-R interval (CVRR\%) of an ECG. This time, we examined the reliability of $\mathrm{CVaa} \%$ as the index of heart rate variability. The purpose of this study is to evaluate the validity of the APG system to estimate heart rate variability, and to introduce the APG system as a system which would estimate autonomic nerve function instead of an ECG.

\section{METHODS}

\section{Instrument}

An APG machine named ARTETT (U-MEDICA, Inc. Co., Osaka) was used for this study (Fig. 1). ARTETT's sensor is an infrared sensor of the reflected type. The center wavelength is $940 \mathrm{~nm}$, and the output maximum amplitude is $\pm 3.3 \mathrm{~V}$ in the amplification circuit. The time constant of the direct current cut is 1.5 seconds. The sampling frequency for the analog to digital exchange is $1,000 \mathrm{~Hz}$, which corresponds to the standard of an ECG. The resolution ability is $3.23 \mathrm{~m} \mathrm{~V} /$ digit. ARTETT can be connected to a computer by USB Ver1.1. The USB cable is an A-B type whose length is $1.5 \mathrm{~m}$. The filter treatment is done through the software. Power supply frequency removal rate is beyond $60 \mathrm{~dB}$.

ARTETT is equipped with the measurement-and-analyzing software of waveforms. Using this software, an irregular pulse can be automatically removed, and all the a-a intervals (Taa) of waveforms can be measured automatically. The

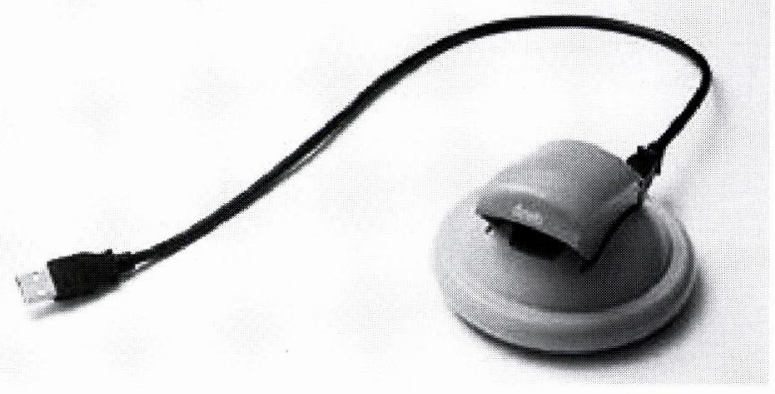

Fig. 1 APG machine (ARTETT; U-medica, Inc., Co.). 
a-peak of the APG corresponds to the starting point of the start of the original pulse wave. From data of Taa, the variation coefficient of the Taa can be calculated automatically.

We show three kinds of waves that were obtained by using ARTETT in Fig. 2, which are the original wave, the first differential wave and the second differential wave (APG). All data of the recorded waveforms can be kept as CSV files in a personal computer. Furthermore, we made a modified ARTETT that has two channels for this research. ECG and APG can be simultaneously measured with this modified ARTETT.

First procedure: Simultaneous measurement of ECG and APG

We simultaneously recorded an ECG and an APG of one subject for 15 minutes by using a 2-channel APG (modified ARTETT) as shown in Fig. 3. The subject was a healthy young man aged 21 . The measurement was taken while he sat in a chair. In Fig. 4, we showed the graph of the comparison of the Taa of an APG and the R-R interval of an ECG. In this graph, we made the position of the R-wave of an ECG align with the a-peak of an APG. Figure 5 shows the difference between the Taa and the R-R interval. Figure 6 shows the simple regression of the Taa and the R-R interval, and the multiple correlation coefficient was calculated.

\section{Second procedure: The APG of 121 healthy people}

The APG waveforms of 121 healthy people were recorded for 2 minutes using an ARTETT in the KS health center in Nagoya from April to May, 2002. The subjects consisted of 20 people of ages $20-29,36$ people of ages $30-39,35$ people of ages $40-49,21$ people of ages $50-59,6$ people of ages $60-69$ and 3 people of ages 70-79. They were all healthy and not any medication. The mean of the variation coefficient with a standard deviation of the Taa in each age category were calculated. The results are shown in Fig. 7.

\section{Third procedure: The APG of 26 diabetics}

The APG waveforms of 26 diabetics were recorded for 2 minutes using an ARTETT at $\mathbf{M}$ hospital in Nagoya in November, 2002. They were outpatients, and were diagnosed with diabetes with the first medical examination. The subjects were 2 people in their 30's, 3 people in their 40's, 11 people in their 50's, 7 people in their 60's and 3 people in their 70's. The mean variation coefficient with the standard deviation of Taa in each age category was calculated. The comparison of healthy person's and diabetic's variation coefficients is shown in the Fig. 8 .

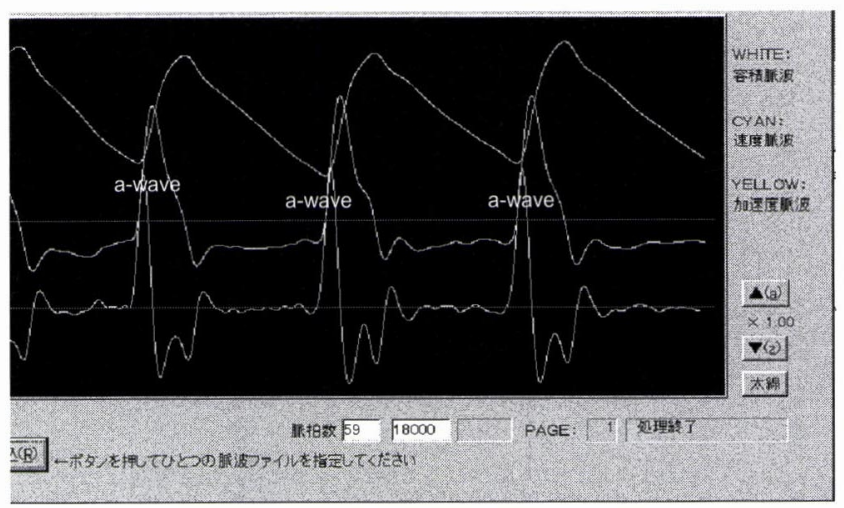

Note. Upper: Digital photopletysmography (DPG) Middle: The first derivative of DPG

Lower: The second derivative of DPG (APG)

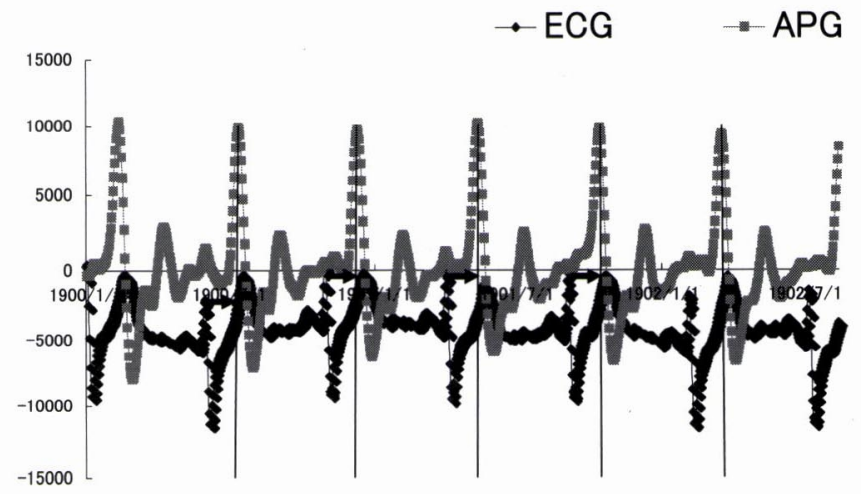

$\rightarrow:$ The fixed delay of the a-wave of an APG

Fig. 3 Simultaneous measurement of ECG and APG.

Fig. 2 The APG waveforms.

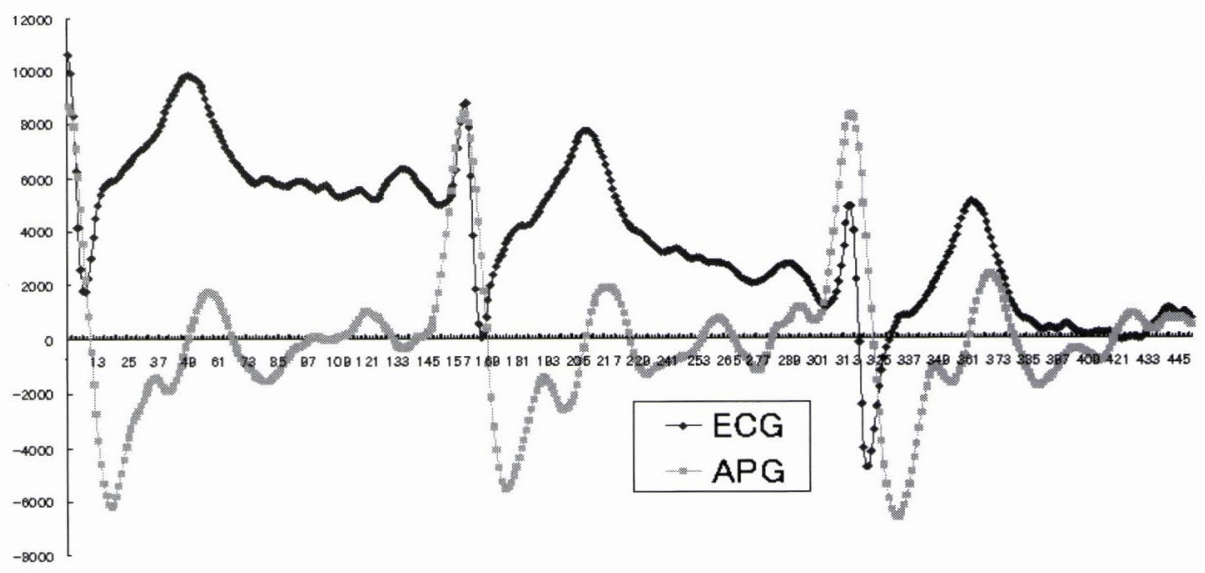

Fig. 4 The graph that the position of R-wave of ECG was made to align with that of a-peak of an APG. 


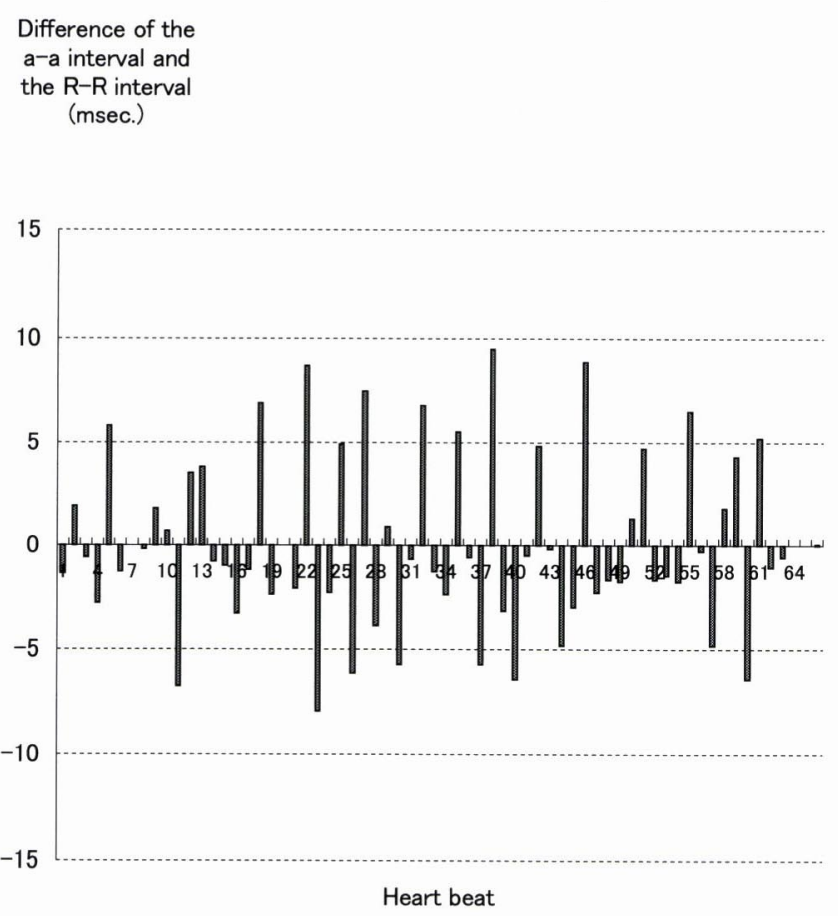

Fig. 5 The difference between the a-a interval and the R-R interval (msec.).

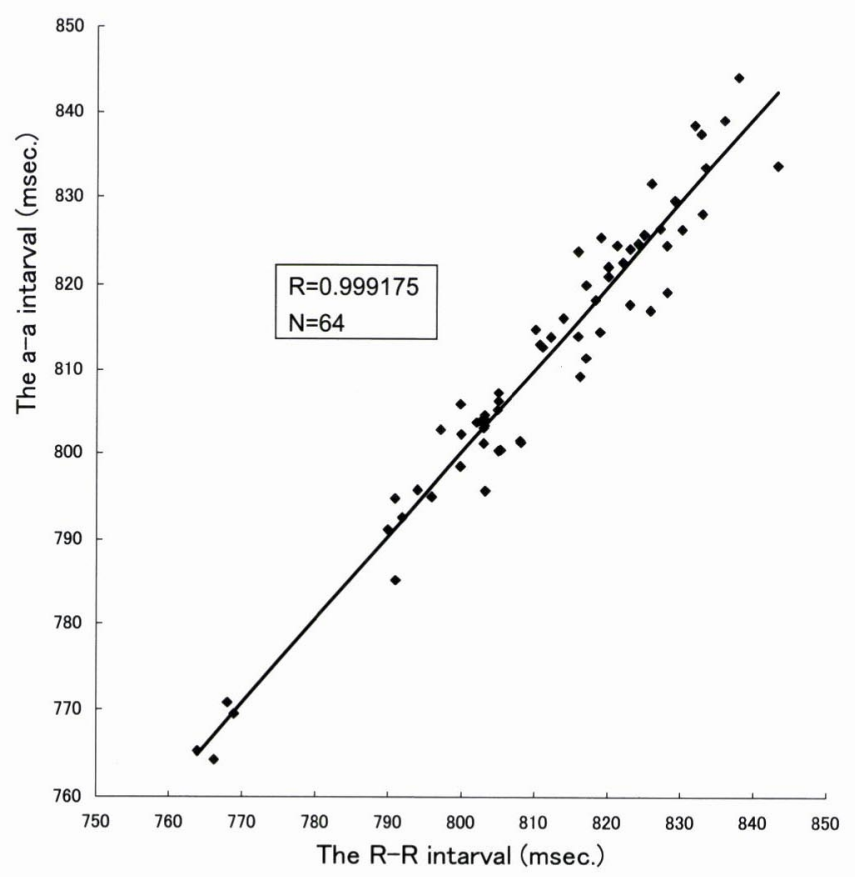

Fig. 6 The simple linear regression of the a-a interval and the R-R interval.

\section{RESULTS}

\section{The Taa of an APG Waveform}

Figure 2 shows three kinds of waves, which are the original wave (DPG), the first differential wave and the second differential wave (APG). An APG waveform has five element waves, which are the positive a-wave, the negative b-wave, the positive c-wave, the negative d-wave, and the positive e-wave. The interval from the a-peak to the next a-peak is called the

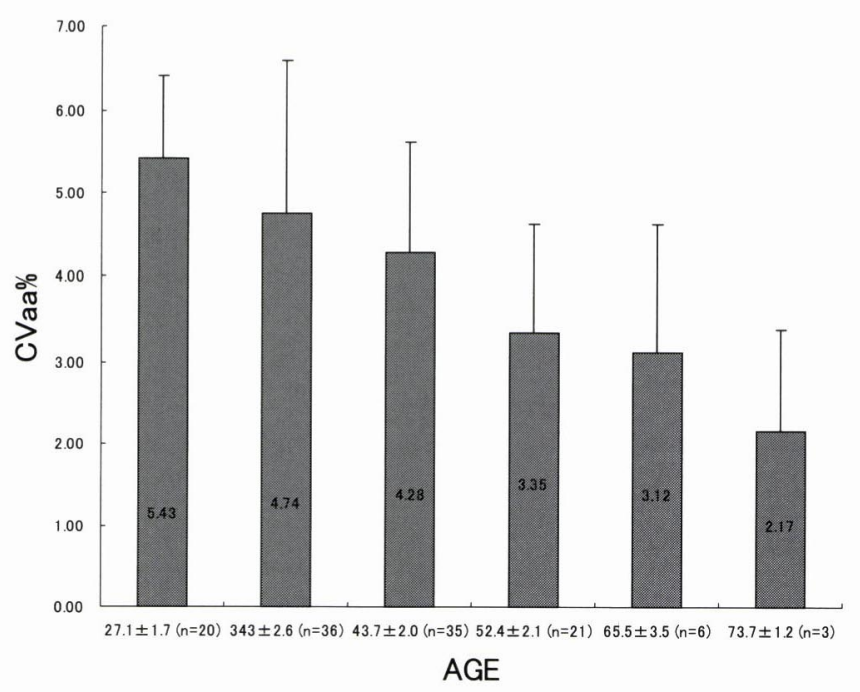

Fig. 7 The average of CVaa\% in healthy people.

CVaa\%

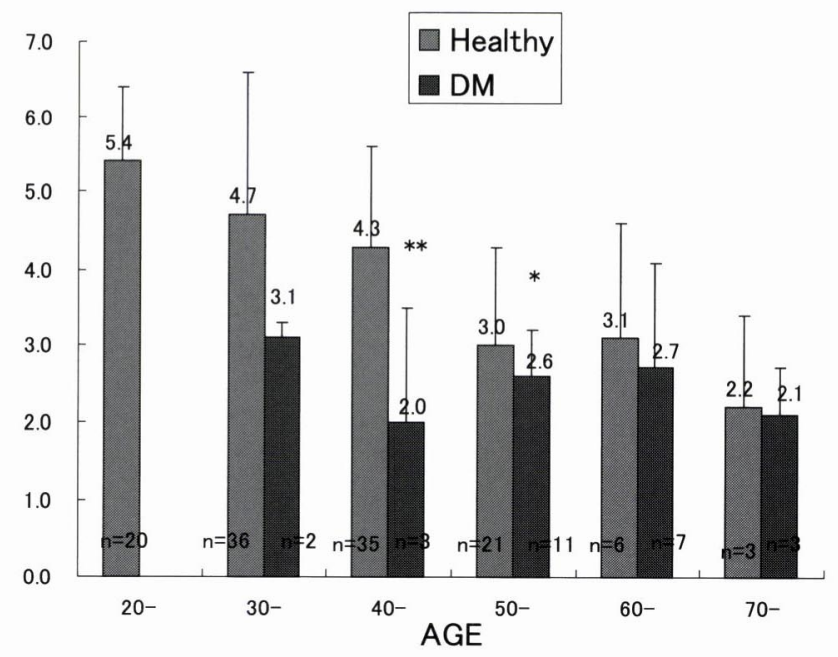

Note: *; $p<0.05,{ }^{* *} ; p<0.01$

Fig. 8 The average of CVaa\% in the diabetics.

Taa. Figure 3 shows an ECG and an APG that were simultaneously recorded. The a-wave of APG appears with the fixed delay after the appearance of the R-wave of an ECG. Then, Fig. 4 is the graph where the first apex of the a-wave of APG aligns with the first apex of the R-wave of an ECG, by adjusting the time delay. It shows that the Taa of APG and the R-R interval on an ECG corresponds. It was also found that a $\mathrm{T}$ wave's end corresponds to an e-wave's end. An APG waveform from the a-peak to an e-wave's end corresponds with the so-called systolic time of an ECG. Figure 5 is the difference between the Taa and the R-R interval. All the differences were within $10 \mathrm{msec}$. Figure 6 showed the simple linear regression of the a-a interval and the R-R interval. The multiple correlation coefficient was 0.999175 . From the results, it was found that an ARTETT is a reliable machine whose sampling frequency is $1,000 \mathrm{~Hz}$. 
Table 1 Previous reports of the CVRR\% of an ECG.

\begin{tabular}{llllllll}
\hline Age group & 10 th. & 20 th. & 30 th. & 40 th. & 50 th. & 60 th. & 70 th. \\
Report 1 & $6.2 \%$ & $6.0 \%$ & $3.7 \%$ & $3.2 \%$ & $3.1 \%$ & $2.8 \%$ & $2.5 \%$ \\
Report 2 & & $4.92 \%$ & $4.02 \%$ & $3.21 \%$ & $2.8 \%$ & $1.58 \%$ & \\
\hline
\end{tabular}

Note. Report 1: Souichirou Mothio: Aging and Disease, IYAKU Journal, p. 182,1993

Report 2: Junko Fujimoto et al.: The Normality Reference Value of the Autonomic Nerve Function and a Standard Prediction-formula, by Using the Variation Coefficient of R-R Intervals on ECG. Diabetes 30: 167-173, 1987

\section{The CVaa\% in healthy persons}

The mean CVaa\% decreased by age as shown in Fig. 7. Each CVaa\% in the age category was $5.4 \%$ with $1.00 \%$ of SD, $4.7 \%$ with $1.87 \%$ of SD, $4.3 \%$ with $1.34 \%$ of SD, $3.4 \%$ with $1.26 \%$ of SD, $3.1 \%$ with $1.49 \%$ of SD, and $2.2 \%$ with $1.23 \%$ of SD. It was clear that the heart rate variability became stable with aging.

Table 1 shows the mean CVRR\% of an ECG in age categories in previous reports. As shown in Fig. 7, the mean CVaa\% was bigger from 0.2 to $1.5 \%$ than the mean CVR-R\% of an ECG in all age categories.

\section{The CVaa\% in diabetics}

Comparing two groups of the healthy and the diabetics, as shown in Fig. 8, both in the age group of 40's and the 50's, mean CVaa\% of the diabetics was significantly lower than that of healthy people $(p<0.01 ; p<0.05$; respectively). In the age group of 40 's, the diabetics' mean CVaa\% was $2.0 \%$ with $1.5 \%$ of SD. In the age group of 50's, the diabetics' mean CVaa\% was $2.6 \%$ with $0.6 \%$ of SD.

On the other hand in the age groups of 30's, 60's and 70's, significant differences were not seen between diabetics and healthy persons. The diabetics' mean $\mathrm{CVaa} \%$ was $3.1 \%$ with $0.2 \%$ of SD in the 30's age group, $2.7 \%$ with $1.4 \%$ of SD in the 60 's age group, and $2.1 \%$ with $0.6 \%$ of SD in the 70 's age group.

\section{DISCUSSION}

We proposed in this research a new method to know the heart rate variability with an APG system. Our new developing machine ARTETT uses the original filter-technology, and carries an analytic algorithm, which is well resistant to the changes in baseline and amplitude (In the patent application). The sampling time of ARTETT is $1,000 \mathrm{~Hz}$, and the performance is trustworthy. Original waveform is reproduced faithfully without distortion (In the patent application).

We confirmed that the CVaa\% which was derived from ARTETT is almost equal to CVRR\% of an ECG. The difference between CVaa\% and CVRR\% in one person was so small we could ignore it. The multiple correlation coefficient was 0.999175 , and the difference time was within 10 msec. It is easily understandable that $10 \mathrm{msec}$ difference is too small to see, when we consider that one mark of the electrocardiogram's paper is $40 \mathrm{msec}$. Even so, the mean CVaa\% would be 0.2 to $1.5 \%$ bigger than the mean CVRR\% of an
ECG in all age categories (See Table 1 and Fig. 7). This difference is considered to be caused by breathing. The Breathing condition must be different in two different postures, which are the sleeping posture of an ECG and the sitting posture of an APG. As for other thinking, a sympatho-vagal balance would be different between the sitting posture and the sleeping posture. Therefore, at any rate, when we want to evaluate the heart rate variability by $\mathrm{CVaa} \%$, the standard value of CVaa\% of each age category is necessary (See Fig. 7).

The significance of knowing the heart rate variability is great. It is said that the decrease in heart rate variability shows mainly in the inhibition of parasympathetic nerve function, although the impairment of the sympathetic nerve also associated with its decline. ${ }^{[3]}$ And, it is known that a decline of heart rate variability shows in aging, too. ${ }^{[4],[5]}$ If we can evaluate the heart rate variability properly, we can gain much information, for example, about circadian rhythm, ${ }^{[6], ~[7]}$ cardiac disorders, ${ }^{[3],[6],[8]}$ neurological disorders, ${ }^{[9],[10]}$ diabetic disorders, ${ }^{[11]-[13]}$ or aging.

In the diabetics of this study, too, the decline of heart rate variability appeared, whether they showed diabetic symptoms such as digestive dysfunction, difficulties in urination, orthostatic hypotension or orthostatic hypertension, or not. This was remarkably seen in the 40's and 50's age groups. Also in this study, we confirmed that heart rate variability presented as CVaa\% became stable with aging. Thus, we could know heart rate variability very easily, and could evaluate it.

In conclusion, the introduced APG system in this study is suitable to estimate heart rate variability. And, when we want to evaluate heart rate variability by CVaa\% of an APG instead of CVRR\% of an ECG, a standard value of CVaa\% in each age is necessary. The APG system has some possibilities for explanation of a mechanism of autonomic-nerve-related diseases.

\section{REFERENCES}

[1] Takada H: Proposal of Aging Score Method by Acceleration Plethysmography. Health Eval Prom 29: 855-861, 2002

[2] Takada H: Acceleration Plethysmography and Aging. KETSUATU 9: 1267-1272, 2002 (In Japanese)

[3] Gulli G, Gemin R, Pancera P, Menegatti G, Vassanelli C, Cevese A: Evidence of Parasympathetic Impairment with Cardiac Syndrome X. Cardiovas Res 52: 208-216, 2001

[4] Mothio S: Aging and Disease. IYAKU J. 1993, p. 182 (In 


\section{Japanese)}

[5] Fujimoto J, et al.: The Normality Reference Value of the Autonomic Nerve Function and a Standard Prediction-formula, by Using the Variation Coefficient of R-R Intervals on ECG. Diabetes 30: 167-173, 1987 (In Japanese)

[6] Manolis AG, Katsivas A, Lazaris E, Kutsogeogis D, Lourvros N: Circadian Modulation of Ventricular Tachycardia Cycle Length Variability in ICD Patients with Dilated Cardiomyopathy. Pacing Clin Electrophysiol 20: 203-207, 1997

[7] Kageyama S: The Variety of R-R Intervals of ECG. NAIKAGAKU 55: 242-246, 1985 (In Japanese)

[8] Carney RM, et al.: Depression, Heart Rate Variability, and Acute Myocardial Infarction. Circulation 104: 2024-2028, 2001
[9] Goth T, Fukushima H, Sasaki G, Matsuo N, Takahashi T: Evaluation of Autonomic Nervous System Functions with Spectral Analysis of Heart Rate Variability in a Case of Tetanus. Brain Dev 23: 791-795, 2001

[10] Yang TF, et al.: Power Spectrum Analysis of Heart Rate Variability in Children with Epilepsy. Childs Nerv Syst 17: 602-606, 2001

[11] Wheeler T, Watkins PJ: Cardiac Denervation in Diabetes. Br Med J 4: 584-586, 1973

[12] De Angelis C, et al.: Modified Autonomic Balance in Offsprings of Diabetics Detected by Spectral Analysis of Heart Rate Variability. Metabolism 50: 1270-1274, 2001

[13] Yoshinari M, Wakisaka M, Nakamura U, Yoshioka M, Uchizono Y, Iwase M: Orthostatic Hypertension in Patients with Type 2 Diabetes. Diabetes Care 24: 1783-1786, 2001 